\title{
Melhoria da resistência ao rasgo de tecido plano através do planejamento de experimentos
}

Gilson Marques Pinheiro

gilsonmarques1@gmail.com

Bruna Caroline Resende Sousa

brunasousa89@hotmail.com

Cássia de Paiva Costa Basílio

cassiadepaiva@hotmail.com

Claudiney Alves da Silva

rcdiney@yahoo.com.br

Geraldo Nilton de Oliveira

professor@geraldonilton.com

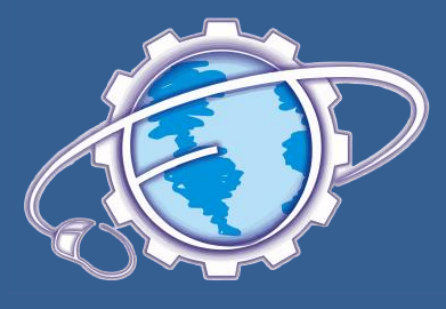

\section{RESUMO}

A resistência ao rasgo é característica de grande importância na indústria têxtil, uma vez que determina a durabilidade e aplicabilidade do tecido. Portanto, tecidos com baixos valores de resistência, que é o caso analisado neste trabalho, geram retrabalhos, segunda qualidade e atraso na entrega, o que afeta o atendimento às necessidades dos clientes e, consequentemente, a competitividade da empresa. Através do levantamento de dados dos processos, constatou-se que o tipo de acabamento aplicado ao tecido influencia diretamente nos valores de resistência ao rasgo. Dessa maneira, através da aplicação da metodologia do planejamento de experimentos (DOE - Design of Experiments), verificou-se o efeito dos diversos produtos das receitas de acabamento do tecido nos valores de resistência ao rasgo. Em decorrência das análises realizadas, foram implantadas alterações naquelas receitas sem, contudo, modificar os fatores de construção do tecido. Os resultados se mostraram significativamente melhores, resultando num aumento médio de 84,4\% nos valores de resistência ao rasgo, eliminando o reprocesso e garantindo o atendimento às especificações.

Palavras-chave: Indústria têxtil, Qualidade, Resistência ao rasgo, Planejamento de experimentos.

\section{Improving resistance to flat-weave fabric ripping through experiment planning}

\begin{abstract}
Resistance to ripping is a characteristic of great importance to the textile industry, since it determines the fabric's durability and usefulness. Thus, fabrics with low degree of resistance, which is the case analyzed in this study, generate reworking, second quality, and delay in delivery, what affects the client's needs for the product and, consequently, the enterprise's competitiveness. Through a survey of the processes' data, it was noted that the kind of the final touch applied to the fabric influences directly on the level of resistance to ripping. Thus, by using the methodology of experiment planning (DOE-Design of Experiments), it was verified the effect that the various products used in the fabric's finishing touch procedures had on the level of rip resistance. In light of the conducted analyses, changes to those procedures were implemented, without altering, however, the factors in the construction of the fabric. The results presented significant improvements, causing an average increase of $84,4 \%$ to the level to rip resistance, eliminating the reprocess and guaranteeing the product's specifications.
\end{abstract}

Key words: Textile Industry; Quality, Resistance to Ripping, Experiment Planning. 


\section{Introdução}

A qualidade na indústria têxtil pode ser garantida através de rigoroso controle, avaliando quão bem seus produtos atendem às especificações estabelecidas pelos clientes. A não execução correta da produção do tecido representa custos adicionais em reprocessos, retalhos e atrasos na entrega, afetando diretamente o atendimento aos clientes.

Entre todas as características determinantes da qualidade do tecido plano acabado, a resistência ao rasgo apresenta-se como importante requisito para a liberação do produto para o cliente final, uma vez que o mercado exige tecidos diferenciados, resistentes e com altos valores agregados.

O rasgo no tecido plano acontece por vários fatores, como tipo de utilização do material têxtil, tipo de esforços a que o material fica exposto e atrito. Diante das várias formas de utilização do tecido, há que se planejar um produto com resistência ao rasgo capaz de garantir segurança e durabilidade.

Os tecidos planos podem ser produzidos com variadas padronagens como sarja, tela, cetim e gorgorão e com gramaturas diferenciadas (variando a densidade dos fios de trama e de urdume), o que interfere diretamente na resistência ao rasgo. Além disso, o tipo de acabamento a que o tecido é submetido influencia, de forma significativa, nos valores de resistência.

Dessa forma, o objetivo deste trabalho foi buscar alternativas que possibilitem aumento da resistência ao rasgo do tecido analisado através da alteração da receita de acabamento sem, contudo, implementar modificações nos fatores de construção, de maneira a elevar os níveis de qualidade do produto e, consequentemente, garantir a satisfação dos clientes.

\section{Resistência ao rasgo de tecidos planos}

O rasgo do material têxtil pode ocorrer por razões diversas, seja pelo tipo de uso a que ele está sujeito, seja por esforço ou força durante o uso ou, mesmo, por rompimento causado por objeto pontiagudo. Cada tecido, conforme a sua estrutura, tem um ponto máximo de resistência ao rasgo. Conhecer esse ponto permite que alterações sejam feitas durante o planejamento da produção, quando maior resistência se faz necessária (BERBERT, 1994).

O rasgo é uma das propriedades responsáveis por acrescentar valor a tecidos submetidos a tensões mecânicas. Um tecido cuja resistência ao rasgo é baixa é considerado produto inferior. Em contraste com a resistência à tração, que envolve a força necessária para romper grande número de fios simultaneamente, a resistência ao rasgo é consideravelmente afetada por características diversas de fios e tecidos, em especial pelo tipo de acabamento a que o tecido é submetido (MUKHOPADHYAY, 2008).

Percebe-se que os tecidos acabados com amaciamento químico apresentam aumento muito elevado na resistência ao rasgo, apesar de outros acabamentos também influenciarem nessa característica, mesmo que de forma relativamente pequena. A direção do rasgo (no sentido da trama ou do urdume) e o material do tecido são fatores que também influenciam nos valores de resistência ao rasgo (MUKHOPADHYAY, 2008).

A determinação da resistência ao rasgo é feita no equipamento denominado dinamômetro, que registra a força (kgf) que uma amostra de tecido suporta a partir de pequeno rasgo já iniciado no corpo de prova. No momento em que a força é aplicada, as garras do dinamômetro separam-se, fazendo que os fios longitudinais deslizem sobre os fios transversais. A estrutura do tecido sofre distorção e, quando um ou alguns desses fios se rompem, registra-se a força desse rompimento (BERBERT, 1994).

O tecido preso ao dinamômetro para a realização do teste de resistência e a distorção da estrutura do tecido quando da aplicação da força podem ser visualizados na Figura 1. 

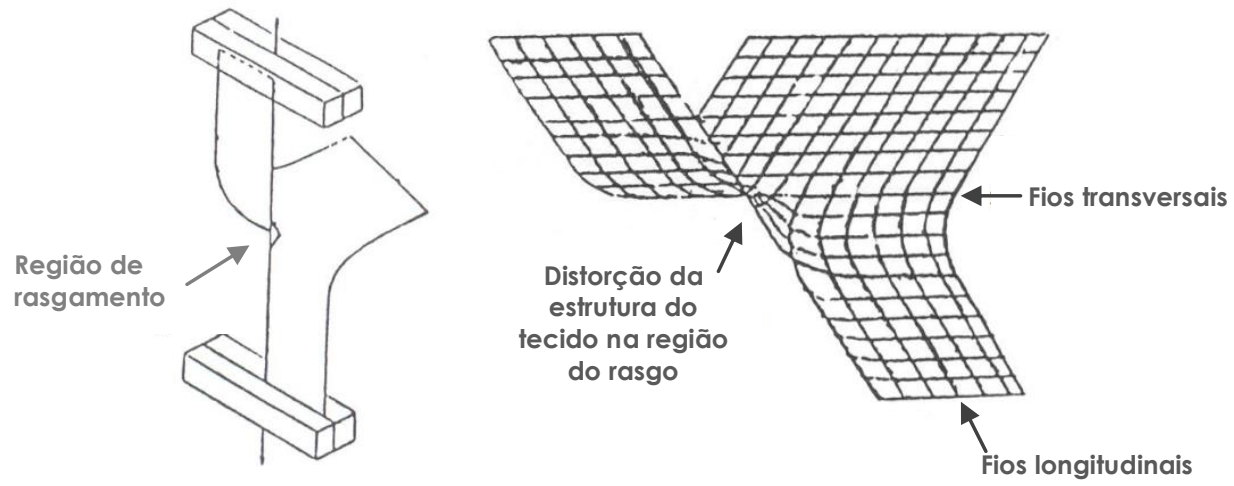

Figura 1 - Realização do teste de resistência ao rasgo e distorção sofrida pelo tecido

Fonte: BERBERT, 1994.

Os procedimentos a serem cumpridos para a correta realização do teste de resistência ao rasgo adotados pela empresa são determinados pela norma ASTM D 2261.

Sendo a resistência ao rasgo do tecido fator determinante para a sua qualidade, esta característica deve ser estudada criteriosamente, e sua perfeita compreensão somente pode ser obtida com uma avaliação conjunta de todas as demais características que a influenciam diretamente.

\section{Planejamento de experimentos}

O planejamento de experimentos, do inglês Design of Experiments (DOE), pode ser definido como metodologia fundamentada em conceitos estatísticos, que objetiva otimizar o planejamento e executar e analisar o experimento (RIBEIRO, 1999 apud MACEDO, 2007).

Um experimento planejado consiste em um teste, ou em uma série de testes, em que se fazem mudanças propositais nas variáveis de entrada de um processo, de maneira que se pode observar e identificar mudanças correspondentes na resposta de saída (MONTGOMERY, 2004).

O DOE permite realizar experiências e avaliar o efeito de muitas variáveis de forma simultânea. Ao usar o DOE, as variáveis $\mathrm{x}$ controladas [...] são modificadas sistemática e simultaneamente, e os efeitos destas mudanças são medidos, modelados e mapeados. O sistema constituído pelas variáveis de controle e as respostas é modelado através de equações estatísticas (DOMENECH, 2002, p. 24).

A aplicação do DOE, aliada ao conhecimento científico disponível e aplicável, fornece ao pesquisador uma compreensão sem igual do processo. O uso dessa técnica, de acordo com a visão de Domenech (2002), proporciona os seguintes benefícios: a) Permite aos pesquisadores entender quais variáveis influenciam na qualidade dos produtos e, dessa maneira, mudá-las ou controlá-las.

b) Possibilita o estudo simultâneo de diversas variáveis, separando seus efeitos.

c) Mostra aos pesquisadores exatamente o que fazer para alcançar as respostas que procuram, possibilitando, assim, diminuição do tempo para o desenvolvimento de um novo produto.

d) Mostra aos integrantes da equipe como o processo funciona, de forma que eles possam incrementar a produtividade, se possível.

e) Permite mapear a relação entre variáveis e respostas, de maneira a conhecer o efeito que as variações nas variáveis de controle têm na resposta.

f) Maximiza a relação custo/benefício dos ensaios, uma vez que o DOE produz a máxima quantidade de informação por ensaio.

g) Representa o processo estudado através de expressões matemáticas.

Um planejamento adequado possibilita o aprimoramento de processos e a redução da variabilidade dos resultados, do tempo de análise e dos custos envolvidos.

Para usar o planejamento de experimentos, é essencial que todos os envolvidos no experimento compreendam seu objetivo, os fatores que devem ser estudados, como o experimento deve ser conduzido e a maneira como os dados devem ser analisados. Montgomery (2004) recomendou, dessa forma, a utilização das seguintes etapas durante a realização de um DOE:

a) Reconhecimento e relato do problema: desenvolver todas as ideias e objetivos específicos do experimento, solicitando entradas de todas as partes envolvidas (engenharia, qualidade, marketing, gerência, cliente e operadores). 
b) Escolha dos fatores e dos níveis: escolher os fatores que devem variar, os intervalos sobre os quais esses fatores variarão e os níveis (valores) específicos em que cada rodada será feita.

c) Seleção da variável resposta: certificar-se de que determinada variável realmente fornece informação útil sobre o processo estudado, de forma a garantir a objetividade da análise dos resultados.

d) Escolha do planejamento experimental: envolve considerar o tamanho da amostra (número de réplicas), a sequência de execução dos ensaios e a necessidade de aleatorização ou do uso de blocos.

e) Realização do experimento: nesta etapa, o monitoramento e o controle do processo são de imensa importância para garantir a validade do experimento.

f) Análise dos dados: fazer o uso de métodos estatísticos, a fim de que as conclusões estabelecidas sejam objetivas, garantindo a confiabilidade e validade dos resultados.

g) Conclusões e recomendações: o experimento deve acarretar soluções práticas sobre os resultados e recomendar um curso de ação. O uso de métodos gráficos permite que se apresentem os resultados, a análise efetuada e futuras repetições do procedimento empregado, se for necessário.

Para o sucesso do experimento, é vital que todos os procedimentos sejam realizados tão bem quanto possível, o que exige conhecimento e comprometimento.

O planejamento de experimentos é, portanto, técnica de extrema importância para a indústria, uma vez que sua aplicação permite resultados mais confiáveis, com economia de dinheiro e tempo, além de suporte ao controle da qualidade, exigências fundamentais em tempos de concorrência tão acirrada.

\section{Metodologia}

Este trabalho é uma pesquisa aplicada que objetivou gerar conhecimentos para a melhoria da resistência ao rasgo de determinado tecido em face da necessidade de atendimento às especificações de clientes. O problema foi abordado de forma quantitativa, à medida que foram utilizados recursos e técnicas estatísticas, como o planejamento de experimentos e histograma, para definir as melhores alternativas de solução.

Nesta pesquisa experimental, a receita de acabamento do tecido foi selecionada como o principal fator que seria capaz de influenciar a resistência do tecido estudado, uma vez que não seria possível a alteração dos fatores estruturais do tecido.

Para o planejamento do experimento foram definidos três fatores e dois níveis, o que corresponde à preparação de oito receitas diferentes. Esses fatores foram definidos através da análise prévia de resultados de testes que tinham como objetivo a verificação da influência de produtos da receita na resistência ao rasgo. Portanto, os produtos da receita de acabamento fatores - são as variáveis de entrada que foram modificadas para se verificarem os efeitos na variável resposta, a resistência ao rasgo.

A amostra de cada receita foi retirada de forma aleatória do tecido preparado durante a sua produção normal, enquanto $\mathrm{o}$ processo de acabamento das amostras ocorreu em laboratório. De cada receita foram realizados ensaios de resistência ao rasgo em quatro corpos de prova.

A análise dos dados foi feita com o auxílio de ferramentas estatísticas, como fluxograma, gráfico de interação, gráfico de efeitos principais e histograma.

\section{Caracterização do problema e ações de melhoria}

\subsection{Definição do problema}

O levantamento dos valores de resistência ao rasgo do tecido analisado indicou resultados muito baixos, especialmente no que se refere ao rasgo de trama, para o acabamento do tecido tinto no processo pad-ox. O tingimento pad-ox é realizado através da impregnação com corantes sulfurosos insolúveis, os quais são reduzidos para aplicação no tecido. Em seguida, o tecido passa pela secagem e, posteriormente, pela oxidação, em que o corante é oxidado para que volte a ser insolúvel no interior da fibra.

O valor de resistência ao rasgo de trama considerado pela empresa como ideal para o produto analisado é de no mínimo 2,0 kgf. Entretanto, com os baixos valores apresentados no acabamento pad-ox, conforme ilustrado no Gráfico 1, adota-se o valor de referência de 1,2 kgf e, mesmo considerando esse valor, o atendimento a pedidos de clientes apresenta-se comprometido. 


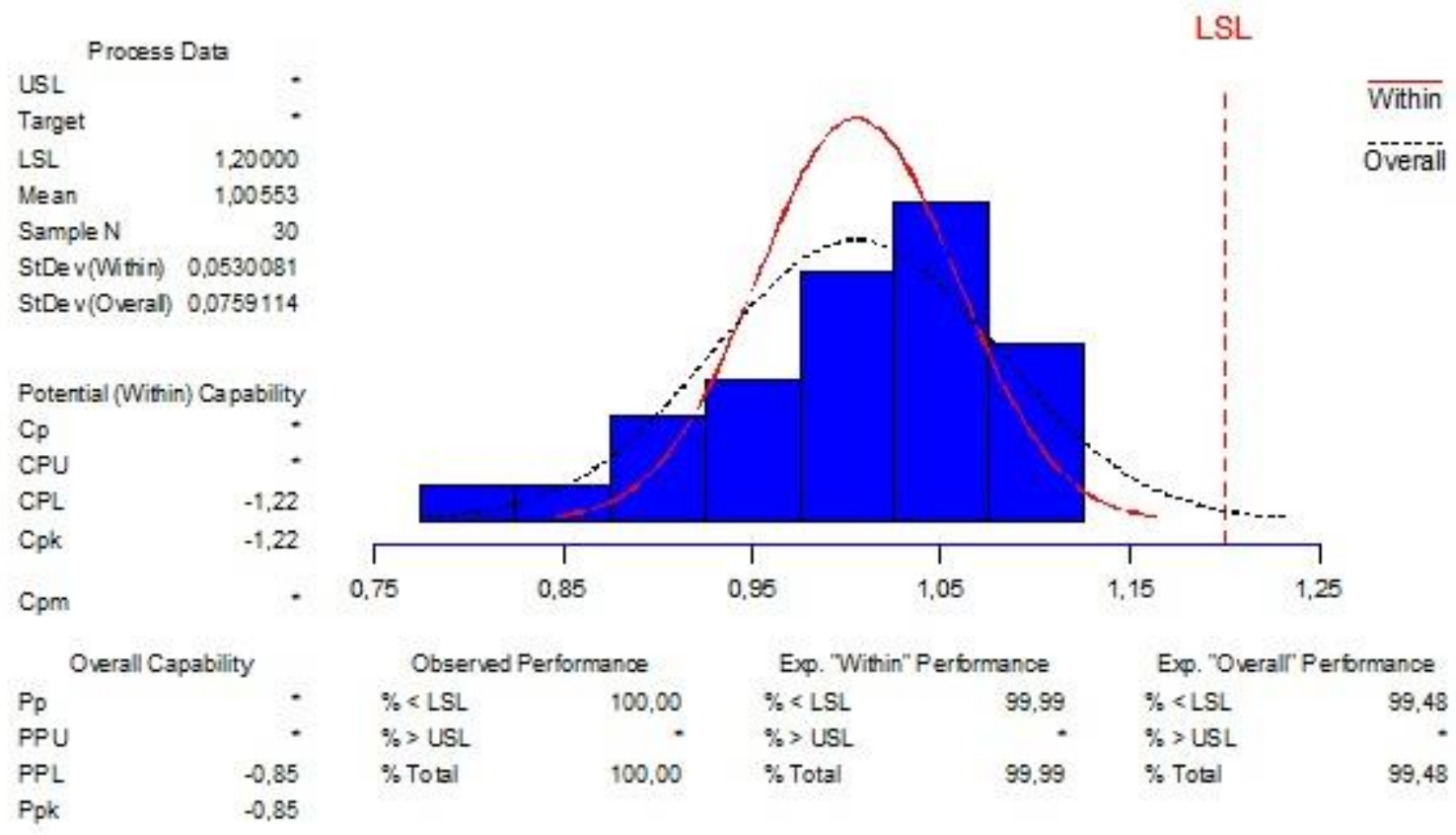

Gráfico 1 - Valores de resistência ao rasgo de trama no acabamento pad-ox

Analisando os resultados de resistência ao rasgo do tecido analisado em outro acabamento para o tecido tinto no processo reativo -, observa-se que os valores se encontram próximos do limite desejável, conforme apresentado no Gráfico 2. O tingimento reativo é obtido por impregnação com corante reativo solúvel em água, que em meio alcalino reage com a celulose, formando uma ligação química entre ele e a fibra. O tecido já impregnado com o corante e álcali e permanece em repouso por tempo necessário para que ocorra a reação química. Por fim, o tecido é lavado para eliminação de resíduos do tingimento e do corante que não reagiu com a fibra.

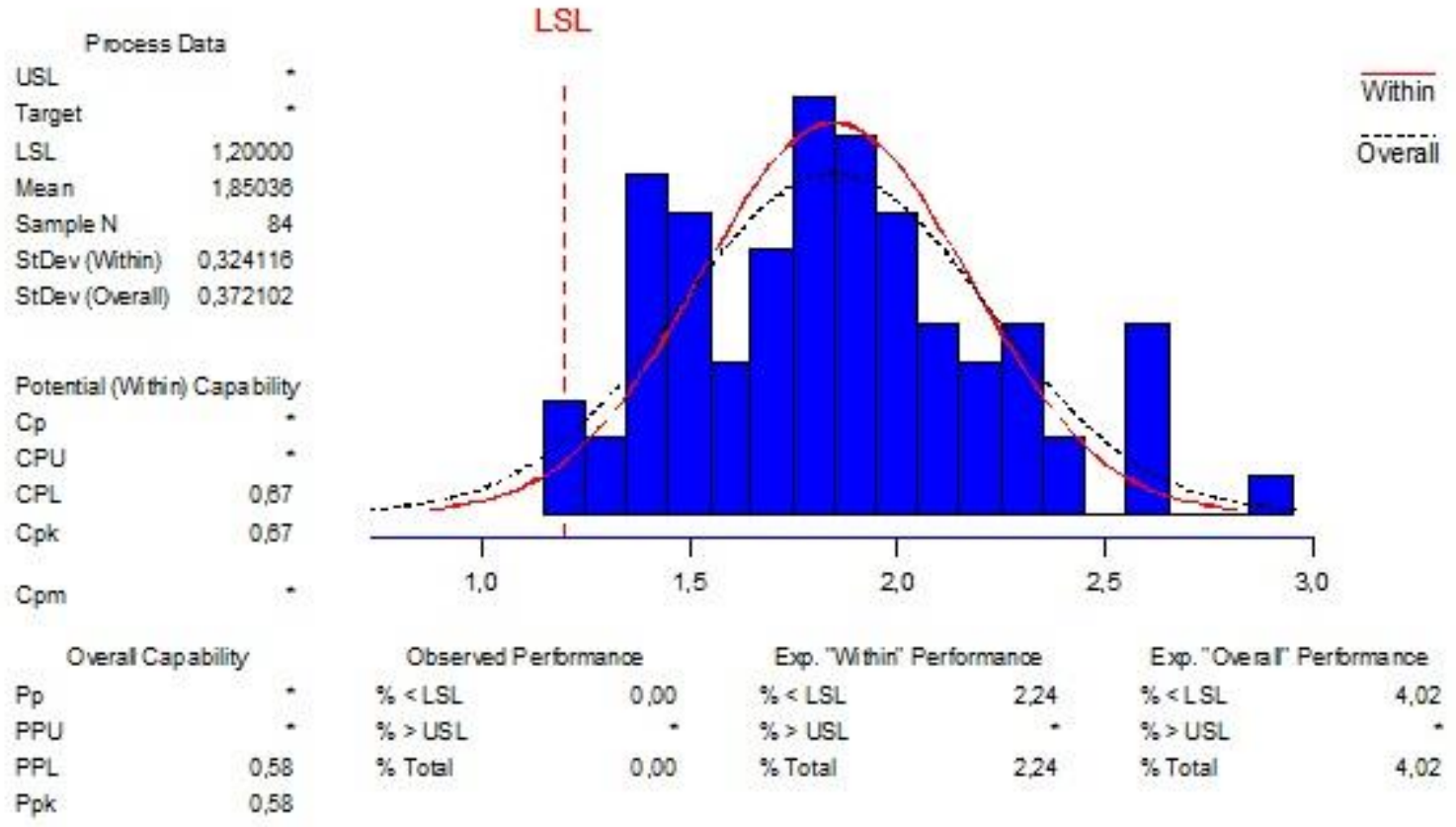

Gráfico 2 - Valores de resistência ao rasgo de trama no acabamento reativo 


\section{2 - Análise do problema}

Percebe-se que os valores de resistência ao rasgo de trama do tecido analisado, quando este é beneficiado no acabamento reativo, são superiores aos obtidos no acabamento pad-ox. Nesse sentido, foram analisados os fluxos produtivos referentes a estes dois tipos de acabamento (Figura 2), sobretudo no que diz respeito às receitas utilizadas.

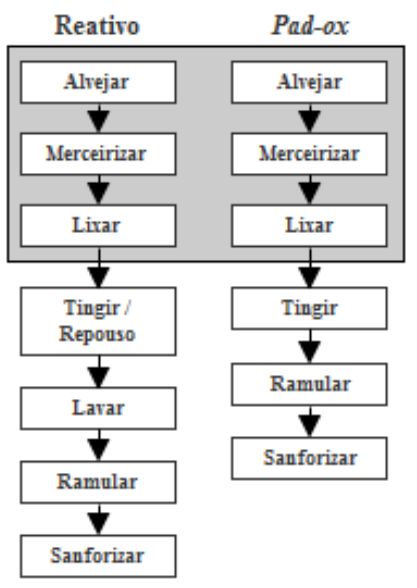

Figura 2 - Processos de manufatura do tecido analisado

Analisando as receitas dos banhos de acabamento que os tecidos recebem, verificou-se que os produtos e suas concentrações são diferentes, conforme descrito na Tabela 1.
Tabela 1 - Receitas dos banhos de acabamento

\begin{tabular}{|c|c|c|}
\hline \multirow{2}{*}{ Produtos } & \multicolumn{2}{|c|}{ Concentração [g/l] } \\
\cline { 2 - 3 } & Reativo & Pad-ox \\
\hline X & 4 & - \\
Y & 15 & - \\
Z & 10 & 40 \\
W & 3 & - \\
K & 1 & - \\
T & 20 & - \\
\hline
\end{tabular}

Com o objetivo de confirmar se os altos valores de resistência encontrados no tecido estudado com acabamento reativo são provenientes da receita do banho de acabamento, foi processada pequena metragem desse tecido no fluxo pad-ox com a receita de banho reativo. Após o acabamento, foi retirada uma amostra para a realização do teste de resistência ao rasgo de trama, cujo resultado foi de 2,0 kgf, valor muito superior à média dos valores de resistência ao rasgo do tecido no fluxo pad-ox, que é de 1,0 kgf, conforme demonstrado no Gráfico 1.

Em razão do ótimo resultado apresentado neste teste, planejou-se um segundo experimento laboratorial com o objetivo de identificar qual produto da receita do banho reativo é responsável pelo acréscimo de resistência. Para realização desse experimento, foram produzidas sete receitas, com os mesmos produtos e concentrações utilizados no banho reativo, porém eliminando a cada receita um produto, conforme descrito na Tabela 2.

Tabela 2 - Planejamento das receitas de banho para o experimento em laboratório

\begin{tabular}{|c|c|c|c|c|c|c|c|}
\hline \multirow{2}{*}{ Produtos } & \multicolumn{7}{|c|}{ Concentração [g/l] } \\
\cline { 2 - 8 } & Receita 1 & Receita 2 & Receita 3 & Receita 4 & Receita 5 & Receita 6 & Receita 7 \\
\hline X & 4 & - & 4 & 4 & 4 & 4 & 4 \\
Y & 15 & 15 & - & 15 & 15 & 15 & 15 \\
Z & 10 & 10 & 10 & - & 10 & 10 & 10 \\
W & 3 & 3 & 3 & 3 & - & 3 & 3 \\
K & 1 & 1 & 1 & 1 & 1 & - & 1 \\
T & 20 & 20 & 20 & 20 & 20 & 20 & - \\
\hline
\end{tabular}

Os resultados dos testes de resistência ao rasgo de trama obtidos no experimento encontram-se no Gráfico 3.

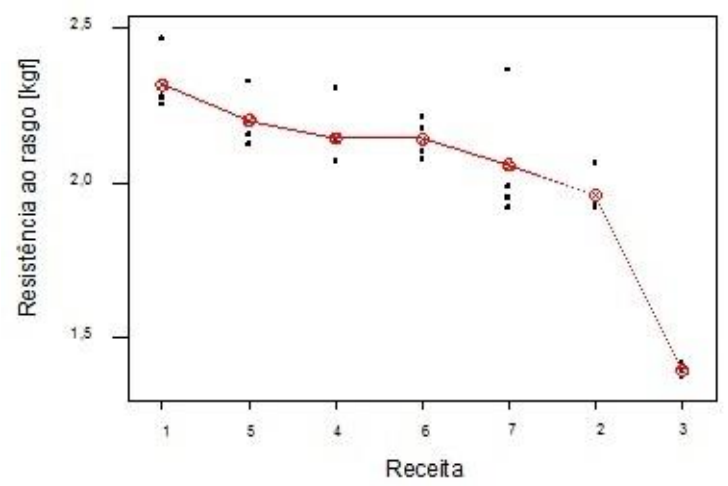

Gráfico 3 - Resistência ao rasgo de trama, com diferentes receitas de acabamento reativo 
Observa-se, através do Gráfico 3, que, ao retirar o produto $\mathrm{Y}$ da receita 3 , os valores de resistência ao rasgo de trama sofrem considerável queda. Percebe-se, assim, que esse produto tem grande influência no aumento da resistência ao rasgo de trama do tecido.

Uma vez que o produto Y não está presente na receita do banho de acabamento pad-ox, foi elaborado um plano de ação para estudar o impacto da adição desse produto nesta receita.

$\mathrm{O}$ estudo foi feito através de um planejamento de experimentos - DOE - que consistiu em variar as concentrações dos produtos das receitas de acabamento e, posteriormente, definir a influência e a quantidade mais adequada de cada produto a ser utilizado.

No DOE para a receita de acabamento pad$o x$, descrito na Tabela 3 , acrescentou-se o produto $\mathrm{Y}$ e variaram-se as concentrações deste e do produto $\mathrm{Z}$. $\mathrm{O}$ produto $\mathrm{K}$ foi acrescentado nessa receita devido à sua capacidade de melhorar a absorção do banho de acabamento pelo tecido.

Tabela 3 - Planejamento de experimentos para a receita de acabamento pad-ox

\begin{tabular}{|c|c|c|c|}
\hline \multirow{2}{*}{ Receita } & \multicolumn{3}{|c|}{ Concentração [g/l] } \\
\cline { 2 - 4 } & Produto Y & Produto K & Produto Z \\
\hline 1 & 15 & 0,5 & 30 \\
2 & 25 & 1,5 & 50 \\
3 & 15 & 1,5 & 50 \\
4 & 25 & 1,5 & 30 \\
5 & 25 & 0,5 & 30 \\
6 & 15 & 1,5 & 30 \\
7 & 25 & 0,5 & 50 \\
8 & 15 & 0,5 & 50 \\
\hline
\end{tabular}

Após a preparação do tecido e o planejamento do experimento, foram realizados os testes laboratoriais, conforme as seguintes etapas: no DOE.

a) Preparação do banho conforme definido

b) Impregnação do tecido com o banho no foulard (caixa de impregnação) com pick-up (quantidade de banho absorvida pelo tecido) de $70 \%$.

c) Secagem das amostras a $130{ }^{\circ} \mathrm{C}$ durante 2 $\min$.

d) Preparação dos corpos de prova para execução do teste de resistência ao rasgo, conforme norma ASTM D 2261/81.

e) Realização dos testes de resistência ao rasgo no dinamômetro Instron modelo 2518 - 107, conforme norma ASTM D 2261/81. f) Análise dos resultados.

$\mathrm{Na}$ análise dos resultados do experimento foram identificados os valores de resistência ao rasgo de trama obtidos a partir de cada receita e a influência dos produtos da receita na resistência ao rasgo, conforme explicitado nos Gráficos 4 e 5, respectivamente.

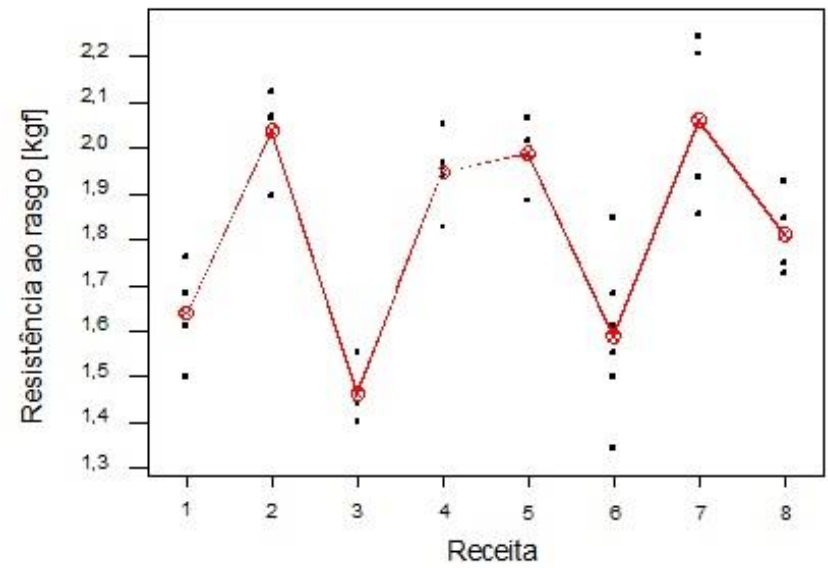

Gráfico 4 - Resistência ao rasgo no acabamento pad-ox, com variação da receita

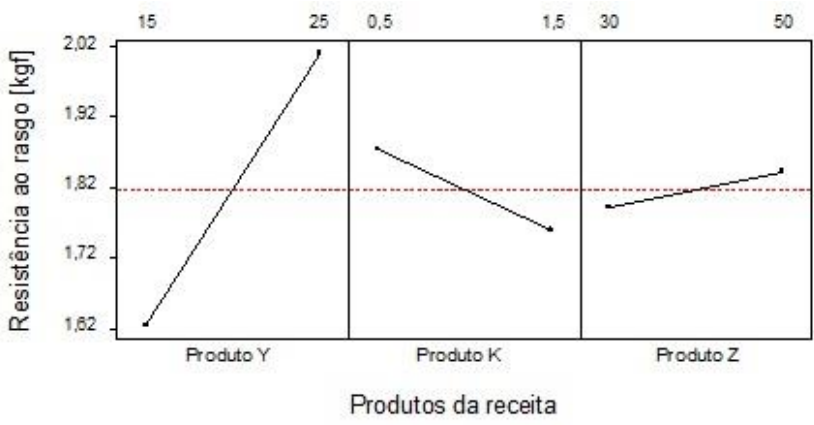

Gráfico 5 - Influência da receita de acabamento pad-ox na resistência ao rasgo de trama

Dessa forma, a equação de regressão para a receita de acabamento pad-ox é definida pela Eq. (1), em que RR designa a resistência ao rasgo e Y, $\mathrm{Z}$ e K representam os produtos da receita.

$$
R R=1,06+0,0385 Y+0,00250 Z-0,115
$$

K

Após a determinação da equação de regressão, foram realizados testes no processo produtivo, comprovando a sua aplicabilidade.

\subsection{Ações implementadas}

Diante da aplicabilidade da equação de regressão, alterou-se a receita pad-ox, com base no fator de incremento da resistência ao rasgo, o produto Y. Analisando os resultados de resistência ao rasgo de trama para as diversas receitas de 
acabamento pad-ox testadas, verificou-se que maiores concentrações do produto $\mathrm{Y}$ melhoram os valores de resistência.

Com os resultados dessas análises, formulou-se uma nova receita a ser aplicada no banho de acabamento pad-ox, conforme indicado na Tabela 4.

Tabela 4 - Receitas do banho de acabamento pad-ox com a adição do produto $\mathrm{Y}$

\begin{tabular}{|c|c|c|}
\hline \multirow{2}{*}{ Produtos } & \multicolumn{2}{|c|}{ Concentração [g/l] } \\
\cline { 2 - 3 } & $\begin{array}{c}\text { Antes do } \\
\text { experimento }\end{array}$ & $\begin{array}{c}\text { Depois do } \\
\text { experimento }\end{array}$ \\
\hline $\mathrm{Z}$ & 40 & 40 \\
$\mathrm{~K}$ & - & 0,5 \\
$\mathrm{Y}$ & - & 25 \\
\hline
\end{tabular}

Foi elaborado um plano de coleta de amostras para o tecido analisado, através do qual se determinou a retirada de uma amostra de todas as ordens de produção desse tecido para realização de testes e monitoramento dos resultados. Dessa forma, possibilitaram-se a identificação de problemas, o levantamento de causas e a implementação de ações para eliminá-las.

\section{Resultados}

Com as novas receitas implementadas no processo de manufatura, a resistência ao rasgo de trama teve acréscimo considerável, conforme demonstrado no Gráfico 6.

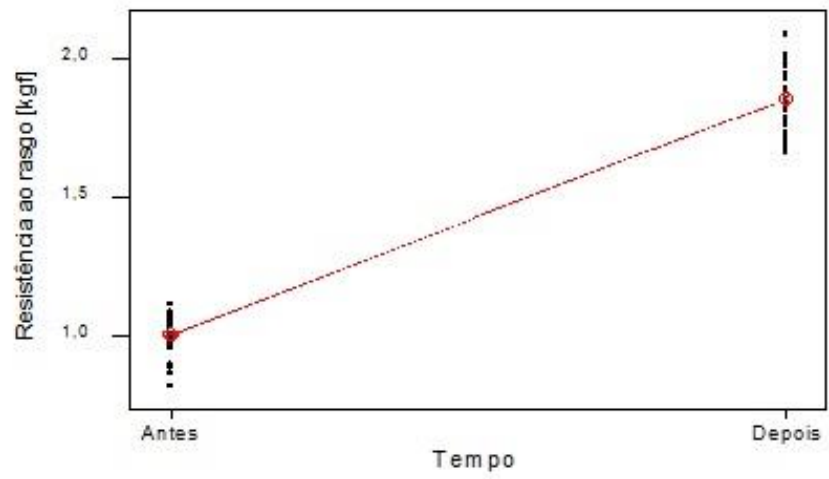

Gráfico 6 - Resistência ao rasgo antes e depois da modificação da receita no acabamento pad-ox

Os valores de resistência ao rasgo de trama se mostram expressivamente melhores, bastante superiores ao valor de $1,2 \mathrm{kgf}$ adotado anteriormente como valor mínimo, conforme explicitado no Gráfico 7.

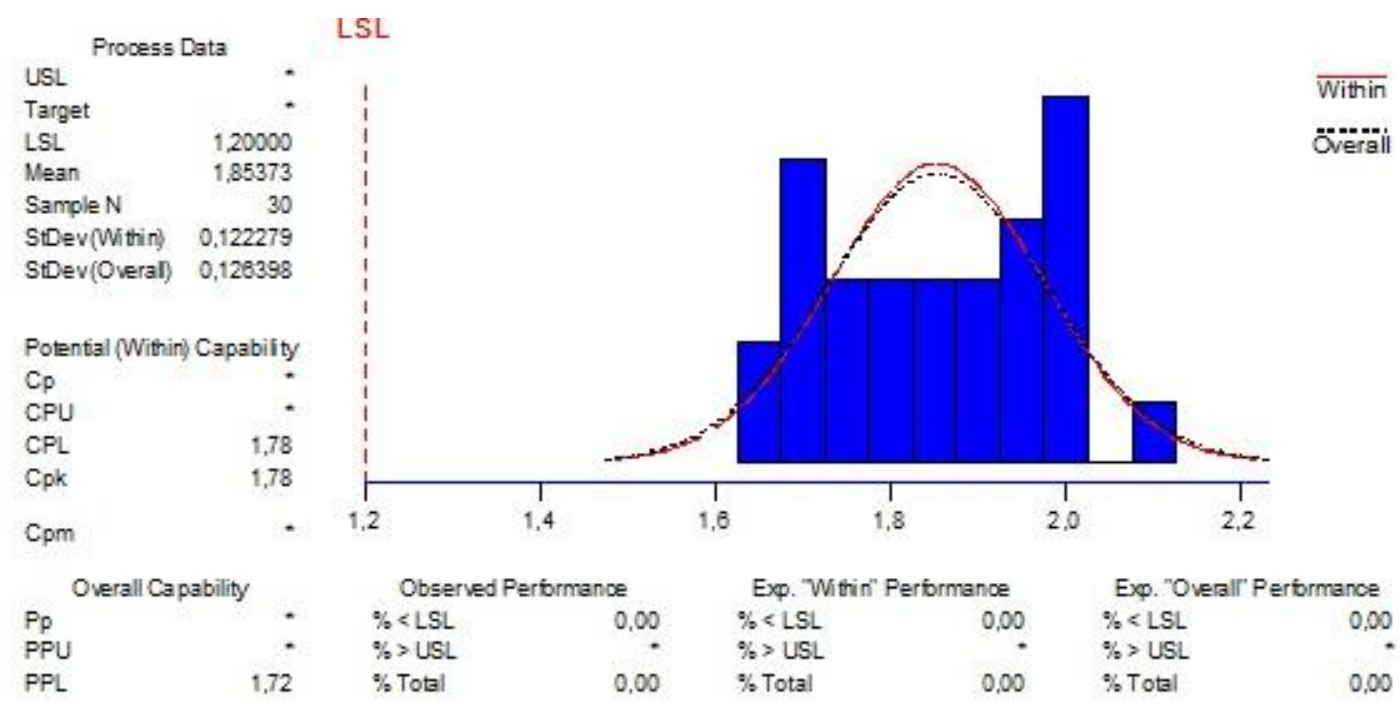

Gráfico 7 - Resistência ao rasgo após a modificação da receita no acabamento pad-ox

\section{Considerações finais}

As análises realizadas neste trabalho confirmaram que os produtos químicos utilizados nos banhos de acabamentos interferem diretamente na resistência ao rasgo do tecido acabado. Essa interferência pode ser positiva ou negativa, dependendo do tipo de produto utilizado e da sua concentração na receita.

Nesta pesquisa, a mudança na receita de acabamento proporcionou melhoria significativa nos resultados de resistência ao rasgo da trama, acarretando aumento médio de $84,4 \%$ nos valores de resistência, o que contribuiu para a redução de reprocesso e garantia do atendimento às especificações.

A utilização de ferramentas estatísticas, como o planejamento de experimentos, permitiu aos pesquisadores entenderem as variáveis que afetam a resistência do tecido e, assim, implementar mudanças no processo de forma a atender às especificações dos clientes. Além disso, o uso 
adequado de métodos estatísticos possibilitou a geração de conhecimentos importantes sobre o processo, contribuindo para a melhoria da memória tecnológica da empresa.

\section{Referências}

BERBERT, Marlene Militão da Silva. A resistência ao rasgamento avaliada em tecidos planos. Textília, Rio de Janeiro, p. 30-36, 1994.

DOMENECH, Carlos. Estratégia 6 o: Etapa Melhorar. M.I. Domenech Consultores. [S.1. : s.n.t.]: Versão 3, 2002.

MACEDO, Rose M. P. R. de. Aplicação da metodologia de planejamento de experimentos para formulação de massas cerâmicas para telhas. 2007. 117 f. Tese (Doutorado em Ciência e Engenharia de Materiais) -Universidade Federal do Rio Grande do Norte, Natal, 2007. Disponível em: $<$ http://ftp.ufrn.br/pub/biblioteca/ext/bdtd /RoseMPRM.pdf>. Acesso em: 12 set. 2010.

MONTGOMERY, Douglas C. Introdução ao controle estatístico da qualidade. 4. ed. Rio de Janeiro: LTC, 2004.

MUKHOPADHYAY, Arunangshu. Impact of functional finishes on tearing strength of fabrics. Melliand International, Germany, p. 172-174, 2008.

Artigo selecionado entre os 10 melhores do VII Encontro Mineiro de Engenharia de Produção EMEPRO 2011. 